\title{
Arsenic-Associated Changes to the Epigenome: What Are the Functional Consequences?
}

\author{
Kathryn A. Bailey • Rebecca C. Fry
}

Published online: 19 January 2014

(C) The Author(s) 2014. This article is published with open access at Springerlink.com

\begin{abstract}
Inorganic arsenic (iAs) poses a major threat to worldwide human health, and yet the molecular mechanisms underlying the toxic effects associated with iAs exposure are not well understood. There is increasing experimental evidence indicating that epigenetic modifications may play a major role in the development of diseases associated with exposure to environmental toxicants. Research in the field has firmly established that iAs exposure is associated with epigenetic alterations including changes in DNA methylation, miRNA abundance, and posttranslational histone modifications. Here, we summarize recent studies that have expanded the current knowledge of these relationships. These studies have pinpointed specific regions of the genome and genes that are targets of arsenical-induced epigenetic changes, including those associated with in utero iAs exposure. The recent literature indicates that iAs biotransformation likely plays an important role in the relationship between iAs exposure and the epigenome, in addition to the sex and genetic background of individuals. The research also shows that relatively low to moderate exposure to iAs is associated with epigenetic effects. However, while it is well established that arsenicals can alter components of the epigenome, in many cases, the biological significance of these alterations remains unknown. The manner by which these and future studies may help inform the role of epigenetic modifications in the development of iAs-associated disease is evaluated and the need for functional validation emphasized.
\end{abstract}

\footnotetext{
K. A. Bailey $\cdot$ R. C. Fry $(\bowtie)$

Department of Environmental Sciences and Engineering, UNC Gillings School of Global Public Health, University of North Carolina at Chapel Hill, Campus Box 7431,

Chapel Hill, NC 27599, USA

e-mail: rfry@unc.edu
}

Keywords Arsenic $\cdot$ DNA methylation $\cdot$ Histone post-translational modifications $\cdot$ MicroRNAs $\cdot$ Epigenetic reprogramming $\cdot$ Prenatal exposure $\cdot$ Epigenome

\section{Introduction}

Exposure to arsenic is a major global threat to human health. The most common route of exposure worldwide is through the consumption of drinking water contaminated with naturallyoccurring, geologic sources of inorganic arsenic (iAs) [1]. Although the World Health Organization (WHO) has recommended that levels of iAs in drinking water should not exceed $10 \mathrm{ppb}$, it is estimated that over 100 million people worldwide are exposed to iAs levels in drinking water that are considered detrimental to human health $[2,3]$. Numerous chronic conditions, such as cardiovascular and peripheral vascular disease, diabetes mellitus, neurological effects, and cancers of the skin, lung, liver, urinary bladder and prostate, may develop after long-term iAs exposure [4]. Much remains to be learned about the factors that influence susceptibility of chronically-exposed individuals to these varied adverse health effects, but they likely include individual genetic background, nutritional status, toxicant co-exposure, the dose and duration of iAs exposure, and capacity of individuals to biotransform iAs to reactive metabolites [5, 6].

The molecular mechanisms underlying the diverse health effects associated with iAs exposure are not well understood and are likely complex. Numerous toxic effects have been attributed to exposure to iAs and its reactive metabolites. For instance, the generation of reactive oxygen species (ROS) is believed to play a major role in mediating the toxic effects of various arsenicals by causing biomolecular damage and the activation of cellular signaling pathways that promote disease development [7]. However, as no single mechanism has been identified to date as a key event in the development of any 
iAs-associated disease, it is plausible that several mechanisms are involved, possibly acting sequentially, simultaneously, and/or synergistically.

In recent years, there has been increased recognition of the importance of the epigenome in maintaining cellular homeostasis, and therefore it has been hypothesized that environmentally-induced epigenetic perturbations may play an important role in disease development. Detailed descriptions of the various components of the epigenome are outside the scope and purpose of this work but are the subjects of reviews elsewhere $[8,9]$. Briefly, the epigenome refers to potentially heritable biological information contained outside the DNA sequence that functions as regulators of gene function [8-10]. The most extensively-studied components of the epigenome include DNA methylation and covalent histone post-translational modifications (PTMs), which regulate gene expression at the transcriptional level. In recent years, microRNAs (miRNAs) have been increasingly recognized as another component of the epigenome. As they regulate mRNA levels primarily at the post-transcriptional level and as they act "above the genome" to control gene expression, they are included in this review. Through their roles in regulating gene expression, each of these components therefore plays a critical role in maintaining cellular homeostasis by interacting in coordinated responses to regulate organismal development, maintain genetic stability, and mediate responses to environmental stressors [11-13].

The recognition that epigenetic changes may mediate the toxic effects of iAs stems in part from the observation that it is a potent carcinogen but not a point mutagen. The long-term health consequences of prenatal iAs exposure also support that iAs may exert adverse effects via epigenetic mechanisms. In both humans and animal models, exposure to iAs in utero is associated with increased risk for chronic disease development later in life [14-16]. In animal models, experimental evidence suggested this altered disease susceptibility was not associated with changes in DNA sequence, but instead, persistent changes in the expression and epigenetic landscape of critical genes, including those implicated in chronic disease development [17, 18]. Observations such as these are in line with the hypothesis that environmental influences in utero play a major role in the fetal origins of adult disease by causing permanent changes, or "reprogramming," of the epigenome in the fetal environment that persist throughout life $[13,19]$. These epigenetic changes are hypothesized to cause altered disease risk via reprogramming the expression patterns of key genes such as silencing tumor suppressors. Importantly, these altered epigenetic marks can be metastable, and thus the effects of altered gene expression and disease risk have the potential to be transgenerational $[20,21]$. Therefore, the association between prenatal iAs exposure and the development of a wide variety of chronic diseases in adulthood is consistent with iAs acting as a prenatal toxicant with widespread epigenetic effects. Various arsenic species have been shown to have effects on the epigenetic landscape outside the fetal environment as well. As recently reviewed, some of these epigenetic effects have been implicated as critical factors in the development of diseases associated with chronic iAs exposure [22].

As the effects of arsenicals on the epigenome have been the subjects of several reviews in recent years [22-24], the purpose of the present review is to provide a critical evaluation of the major trends in the more current literature (2011-2013) in which the relationship between arsenic exposure and the epigenome has been examined. Previous reviews have emphasized the following general findings associated with arsenical exposure: (1) both global and gene-specific changes in DNA methylation levels have been observed, (2) histones are targeted for post-translational modifications, and (3) miRNA levels are perturbed [22-24]. In the present review, we expand upon this information and synthesize and evaluate the current state of knowledge of arsenic-associated epigenetic modifications, particularly in the context of their biological significance in disease development. While epigenetic perturbations associated with iAs exposure were first reported in the 1980s $[25,26]$, two papers published by the Mass and Waalkes laboratories in 1997 marked the beginning of intensive investigations into the effects of arsenicals on the epigenome [27, 28]. As discussed in more detail below, while knowledge of arsenical-induced epigenetic effects has advanced greatly over the past 16 years, we are very much at the beginning of understanding the functional consequences of these modifications, including the potential for specific modifications to influence disease susceptibility as well as the potential heritability of these effects.

\section{Arsenic and DNA methylation}

In mammals, DNA is methylated almost exclusively at the cytosine residue of CpG-dinucleotides in which a single methyl group is enzymatically transferred from S-adenosyl methionine (SAM) to generate 5'-CpG-3' [29]. CpGs are not distributed evenly throughout the mammalian genome, but instead are clustered into two major regions: (1) $\sim 15 \%$ of CpGs are found in regions of euchromatin known as $\mathrm{CpG}$ islands and $\mathrm{CpG}$ island shores, and (2) $\sim 85 \%$ of $\mathrm{CpGs}$ are found in repetitive interspersed regions of DNA (such as transposable elements) in heterochromatin [30-32]. The best-understood functional consequence of $\mathrm{CpG}$ methylation is gene silencing at the transcriptional level. In general, a negative relationship between promoter $\mathrm{CpG}$ island/CpG island shore methylation and gene expression is observed [33], and CpGs are heavily methylated in transcriptionally-silenced regions of heterochromatin [32]. However, it should be noted the relationship between DNA methylation and gene expression is likely quite complex, as there are numerous instances in which this 
relationship has been positive or no relationship has been observed [33, 34].

To date, DNA methylation is the most extensively studied epigenetic alteration in association with arsenic exposure, which has included in vitro studies, in vivo studies, and studies in human populations (as reviewed in [22-24]). Taken together, studies have revealed that DNA methylation patterns can be altered by iAs and/or iAs metabolites at either the global or gene-specific level. DNA methylation changes have been detected in several genes implicated in iAs-associated disease development, such as promoter hypermethylation of tumor suppressors such as cyclin-dependent kinase inhibitor $2 \mathrm{~A}(C D K N 2 A / p 16)$ or tumor protein 53 (TP53) (as reviewed in $[22,24])$. In addition, global DNA hypomethylation, which has been implicated as a contributing or predisposing factor of carcinogenesis [35], has been observed upon exposure to arsenic species, including cell lines malignantly transformed by iAs in vitro $[22,36,37]$. However, in many cases, the biological consequences of these changes (e.g., corresponding changes in mRNA or protein levels) or significance of relationship (e.g., causal relationship between iAs exposure, DNA methylation alterations, and malignant transformation) have not been established. In addition, specific alterations are not consistently observed across various experimental platforms or studies, which may in part reflect the wide range of arsenic doses and exposure times that have been utilized.

Aside from the examination of the promoter DNA methylation status of genes implicated in iAs-associated diseases, a major research focus has been the investigation of prenatal iAs exposure on DNA methylation patterns. There is considerable experimental evidence suggesting that iAs may act as a prenatal toxicant by altering the DNA methylome. This is supported by the observation that similar relationships between changes in DNA methylation patterns and altered disease risk has been observed with exposure to other prenatal toxicants or perturbed prenatal nutritional status [38-42].

Another major area of interest has been the investigation of the relationship between iAs biotransformation and the DNA methylome. IAs biotransformation involves a series of alternating reduction and oxidative methylation steps that yields six major arsenicals found in the urine of humans and most mammals, namely trivalent and pentavalent forms of iAs and its monomethylated and dimethylated metabolites (MMAs and DMAs, respectively) [43-45] (Fig. 1). These metabolites vary in their toxicities, and as previously mentioned, the efficiency of iAs biotransformation is believed to be an important factor in the development of iAs-associated diseases, as several iAs metabolites are biologically reactive [6]. As the same methyl donor source, namely SAM, is required for both DNA methylation and iAs biotransformation, it has been hypothesized that iAs biotransformation may influence disease risk by altering DNA methylation levels and therefore influencing gene expression patterns [27, 28].
In recent years, examinations of the relationship between alterations in the DNA methylome and prenatal iAs exposure and/or iAs biotransformation have continued as major research thrusts (Table 1). As in earlier studies, many of these investigations have also examined the DNA methylation profiles of genes implicated in the development of iAsassociated diseases. As discussed below, most of these studies have not focused on mechanisms by which iAs may impact the landscape of DNA methylation, but instead have examined the DNA methylation profiles within the white blood cells of iAsexposed populations, including cord blood of prenatally exposed newborns and peripheral blood isolated from chronically exposed adults.

Our laboratory was interested in identifying genome-wide gene-specific DNA methylation changes associated with chronic iAs exposure in humans. An examination of DNA methylation patterns in peripheral blood leukocytes (PBLs) from an exposed population in Zimapán, Mexico, identified a set of 183 genes with differentially methylated promoters in women with iAs-associated skin lesions vs. women with unlesioned skin [46•]. Most of these genes had promoters that were hypermethylated in women with skin lesions (99\%), and several genes had been associated with iAs-induced diseases such as diabetes mellitus, cardiovascular disease, and cancer. The cancer-associated genes included a "tumor suppressorome" complex containing 17 genes known to be silenced in various human cancers. When these same blood samples were further analyzed in relationship to urinary measurements of iAs, MMAs, and DMAs, distinct patterns of DNA methylation associated with levels of each of these arsenicals were observed [47]. Interestingly, measurements of the percentage of glycosylated hemoglobin in the blood (\%HbAlc) of these women indicated that $94 \%$ were either pre-diabetic or diabetic, and we observed an enrichment of genes with associations with type 1 and type 2 diabetes mellitus associated with one or more of these urinary metabolites. These findings support the role of the different iAs metabolites as factors influencing the epigenome and highlight the need to investigate interindividual differences in iAs metabolism on epigenetic effects. An interesting observation is that many of the identified genes with iAs-associated DNA methylation changes have similar functionality in the cell, and thus likely possess common response elements for transcription factor binding. To explain this phenomenon, the laboratory's current hypothesis is that toxicant-induced transcription factor occupancy influences access of DNA methyltransferases to particular regions of the genome, resulting in "DNA methylation footprints" [48].

Other studies in exposed populations have linked iAs metabolism to differences in DNA methylation patterns, including two studies that have examined the relationship between the DNA methylation patterns and polymorphisms in the arsenic ( +3 oxidation state) methyltransferase $(A S 3 M T)$ gene, 


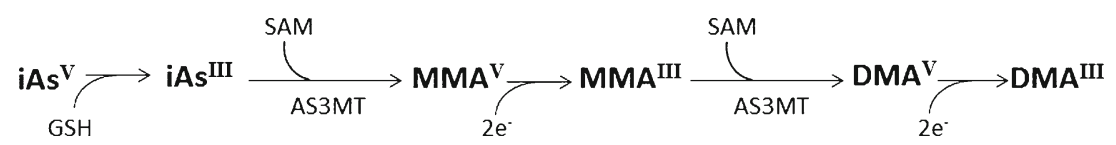

Fig. 1 Arsenic biotransformation in humans. Worldwide, the most prevalent source of arsenic exposure in humans is drinking water contaminated with inorganic arsenic (iAs), which exists in two oxidation states, namely pentavalent arsenate (iAs ${ }^{\mathrm{V}}$ ) and trivalent arsenite (iAs $\left.{ }^{\mathrm{III}}\right)$. IAs ${ }^{\mathrm{V}}$ is reduced to iAs ${ }^{\mathrm{III}}$ mainly in the blood or liver by one of several mammalian enzymes that utilize glutathione (GSSH) or dithiothreitol (DTT) as reductants. The remaining steps of iAs biotransformation, which involve alternating oxidative methylation and reduction steps, occur mainly in the liver. In this process, $\mathrm{iAs}{ }^{\mathrm{III}}$ undergoes an oxidative methylation step catalyzed by arsenic ( +3 oxidation state) methyltransferase (AS3MT) to produce monomethylarsonic acid $\left(\mathrm{MMA}^{\mathrm{V}}\right)$. $\mathrm{MMA}^{\mathrm{V}}$ is subsequently reduced to monomethylarsonous acid (MMA ${ }^{\mathrm{III}}$ ), which is the substrate for

which is the gene encoding the enzyme required for iAs biotransformation (Fig. 1). In chronically-exposed women living in the Argentinian Andes, a major AS3MT haplotype associated with more efficient iAs metabolism was associated with increased methylation of $A S 3 M T$ and differential methylation of several other genes located on chromosome band 10q24 along with $A S 3 M T$ [49]. Similar but weaker trends were also identified in the cord blood of Bangladeshi newborns exposed to arsenic in utero [49].

A separate study examined the relationship between the AS3MT haplotype, concentrations of urinary arsenicals, and DNA methylation levels of the tumor suppressor gene $p 16$, the DNA repair gene mutL homolog 1 , colon cancer, nonpolyposis type 2 (E. coli) (MLH1), and the Long Interspersed Nuclear Element 1 (LINE-1) in the blood of chronically exposed women from the Argentinian Andes [50•]. A significant positive relationship was observed between the slow-metabolizing $A S 3 M T$ haplotype and p16 methylation. The concentration of total urinary arsenic (U-tAs; sum of iAs, MMAs, DMAs) was associated with $p 16$ and MLH1 methylation, urinary \%iAs was positively associated with $p 16$ methylation, and urinary \%DMAs was negatively associated with p16 methylation. Interestingly, although increased urinary ratios of MMAs and/or high ratios of MMAs/DMAs are associated with increased risk for a variety of iAs-associated diseases [6], this study suggests iAs may have more toxic effects than its metabolites on the methylation status of the p16 promoter, at least in DNA isolated from blood. No significant relationship was observed between LINE-1, used as an indicator of global DNA methylation status, and urinary arsenical measurements [50॰].

One study investigated the promoter DNA methylation level of the AS3MT promoter in Native Americans with low vs. moderate U-tAs [51]. Out of $48 \mathrm{CpGs}$ assayed in the $A S 3 M T$ promoter, a region was identified that contained 30 hypomethylated CpGs in the moderate U-tAs group compared to the low U-tAs group, but there were no associations observed between $A S 3 M T$ promoter methylation levels and $\%$ MMAs or \%DMAs in urine. This study also revealed that another round of oxidative methylation and reduction in which dimethylarsinic acid $\left(\mathrm{DMA}^{\mathrm{V}}\right)$ and dimethylarsinous acid $\left(\mathrm{DMA}^{\mathrm{III}}\right)$, respectively, are produced. AS3MT has been shown to catalyze both the oxidative methylation and successive reduction reactions using sadenosyl methionine (SAM) as a methyl donor and various molecules such as nicotinamide adenine dinucleotide phosphate (NADPH), thioredoxin, DTT, or GSH as reductants $\left(2 \mathrm{e}^{-}\right)$[43, 45]. In general, total urinary arsenic in iAs-exposed individuals is composed of 10-20\% total (i.e. trivalent + pentavalent) iAs, $10-20 \%$ total MMAs, and 60-80\% total DMAs [44]. (Modified from: Bailey KA and Fry RC, Arsenicinduced Changes to the Epigenome, in Toxicology and Epigenetics, S.C. Sahu, Editor. 2012, John Wiley \& Sons, Ltd.: Chichester, UK) [22]

short-term exposure of peripheral blood mononuclear cells (PBMCs) to sodium arsenite in vitro induced AS3MT expression, which was accompanied by a decrease in DNA methylation of the $A S 3 M T$ promoter. In addition, $A S 3 M T$ knockdown inhibited arsenite-induced global DNA hypomethylation in PBMCs in vitro. While this study established a relationship between $A S 3 M T$ promoter methylation status and arsenic exposure in humans, further studies will be necessary to determine if these DNA methylation alterations are associated with changes in $A S 3 M T$ expression in human populations and how these changes impact iAs biotransformation and risk of iAs-associated disease development.

Several studies have examined the DNA methylation status of targeted genes including tumor suppressors and/or global methylation of LINE-1 in the cord blood of exposed newborns. Analyses of cord blood from a cohort of newborns in Thailand exposed to varying levels of iAs in utero identified a positive relationship between arsenic exposure and TP53 promoter methylation status but not LINE-1 methylation status [52]. The relationship between DNA methylation patterns of LINE-1 and TP53 and p16 promoters were examined in maternal and umbilical cord leukocytes isolated from iAsexposed mother-child pairs in Bangladesh [53]. Similar trends were observed between DNA methylation trends collected from maternal and newborn blood, in which there was a positive association between maternal U-tAs levels and LINE-1 methylation and some CpG sites within the $p 16$ promoter. The data revealed evidence for a nonlinear doseresponse relationship, as these effects were most evident in the middle tertiles of exposure. These data suggest that moderate levels of iAs, which are likely relevant to many areas endemic for arsenic poisoning, may have significant effects on DNA methylation patterning that could result in genomic alterations.

Supporting the impact of moderate levels of iAs exposure on the epigenome are changes in DNA methylation observed in a population exposed to relatively low levels of iAs. A genome-wide examination of cord blood isolated from newborns from New Hampshire revealed that while no global 


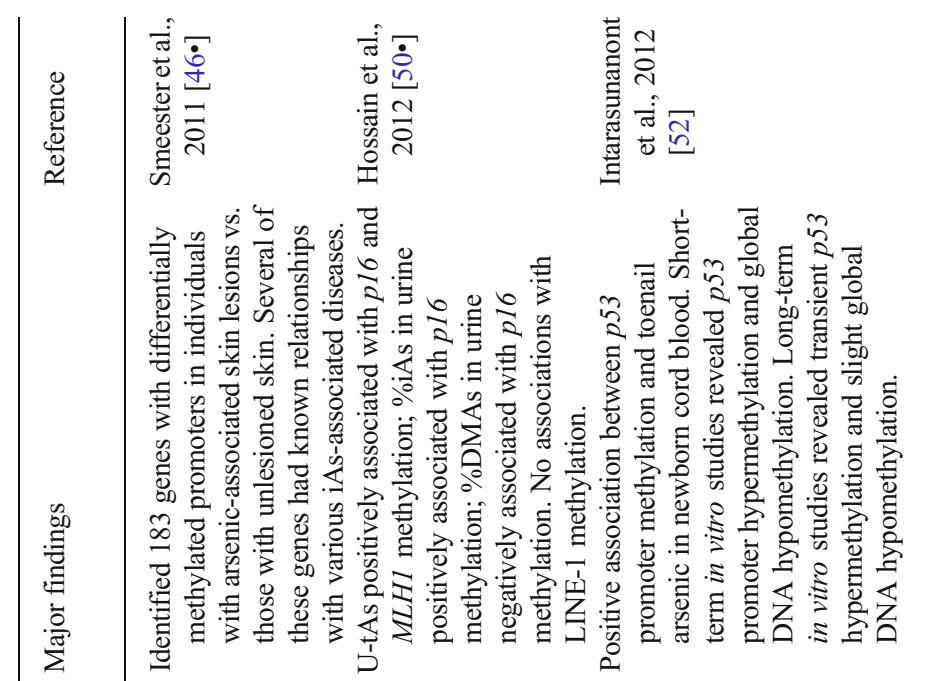

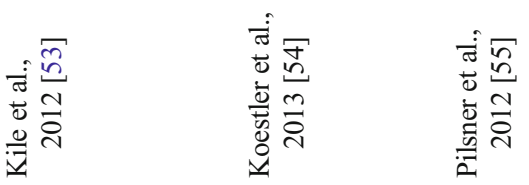

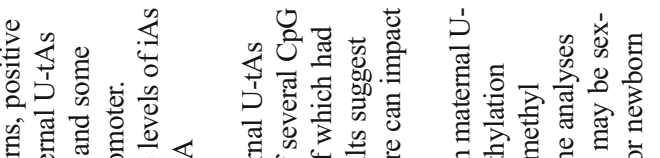

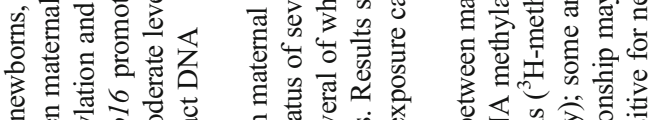

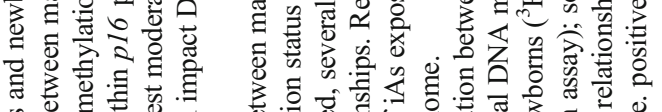

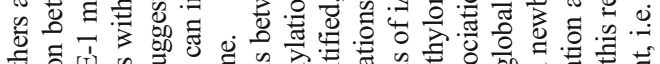

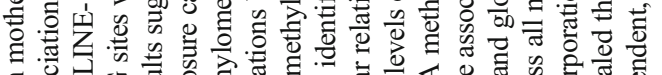
等

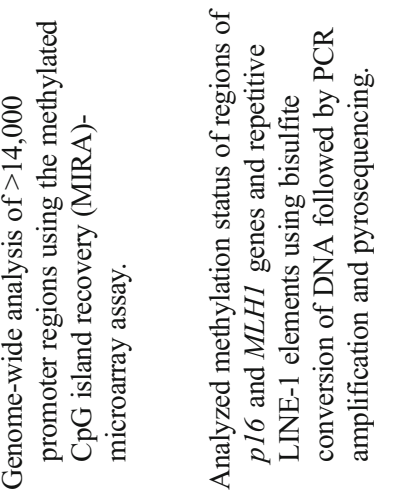

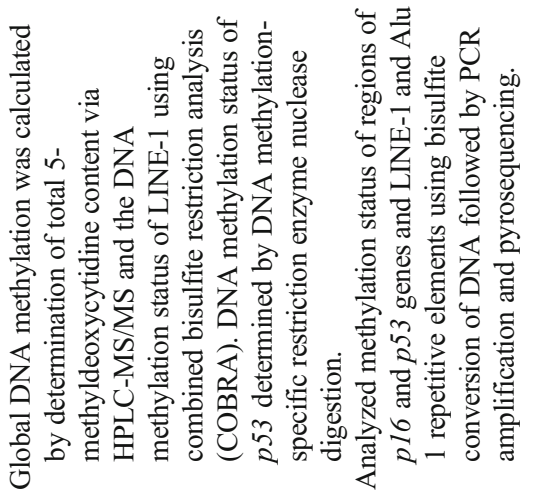

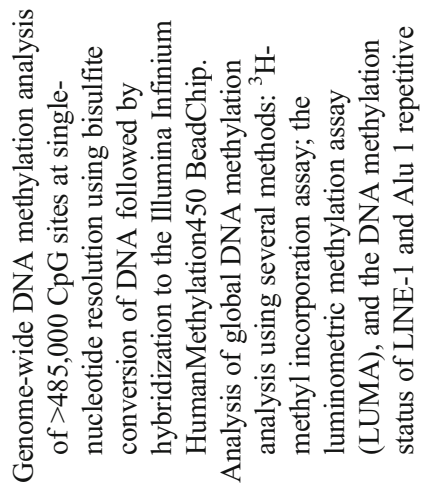
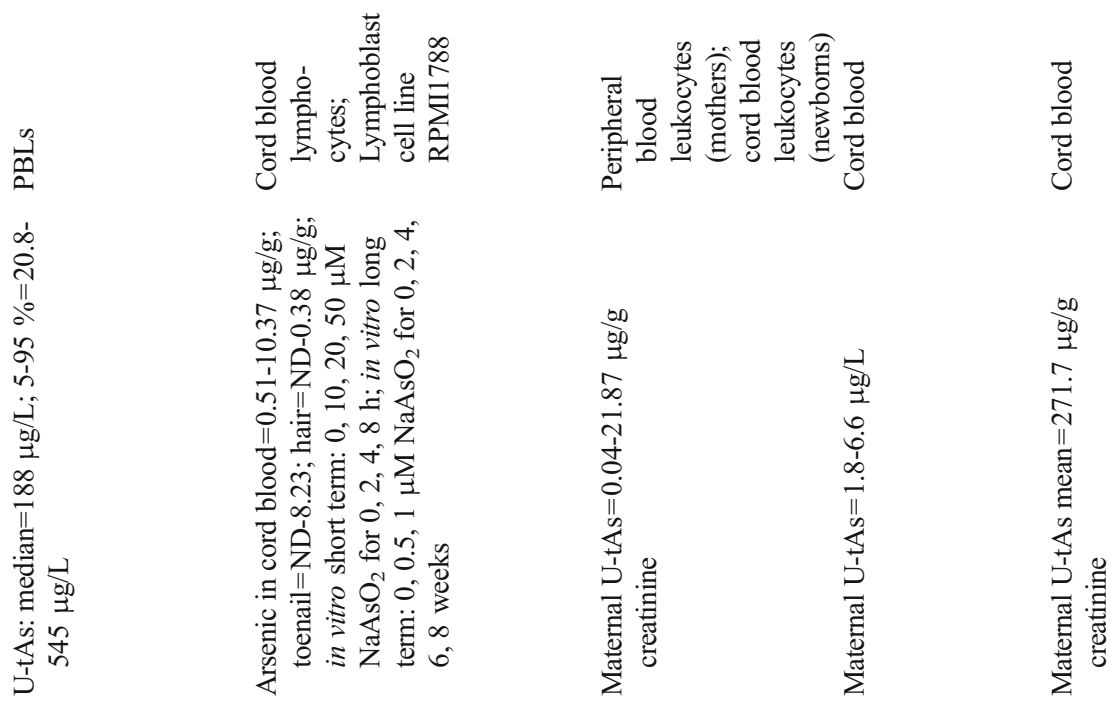

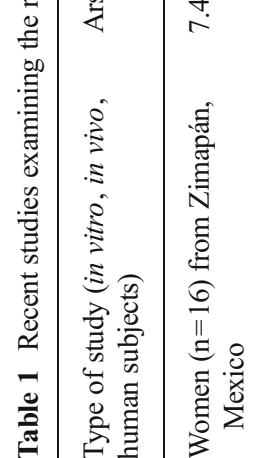

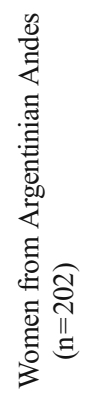
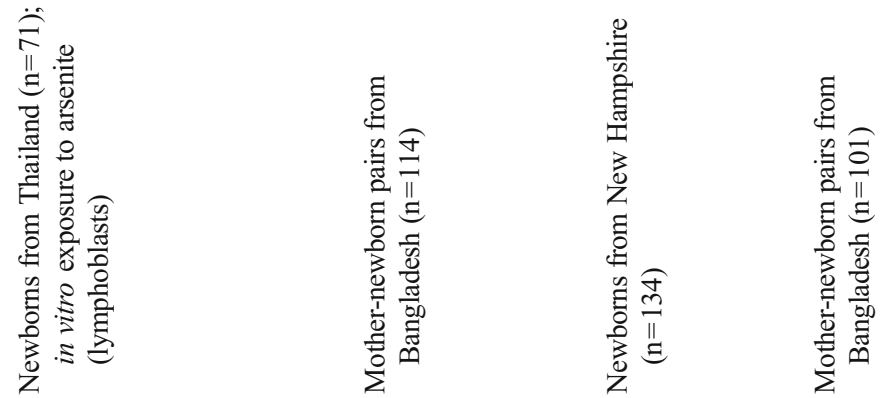


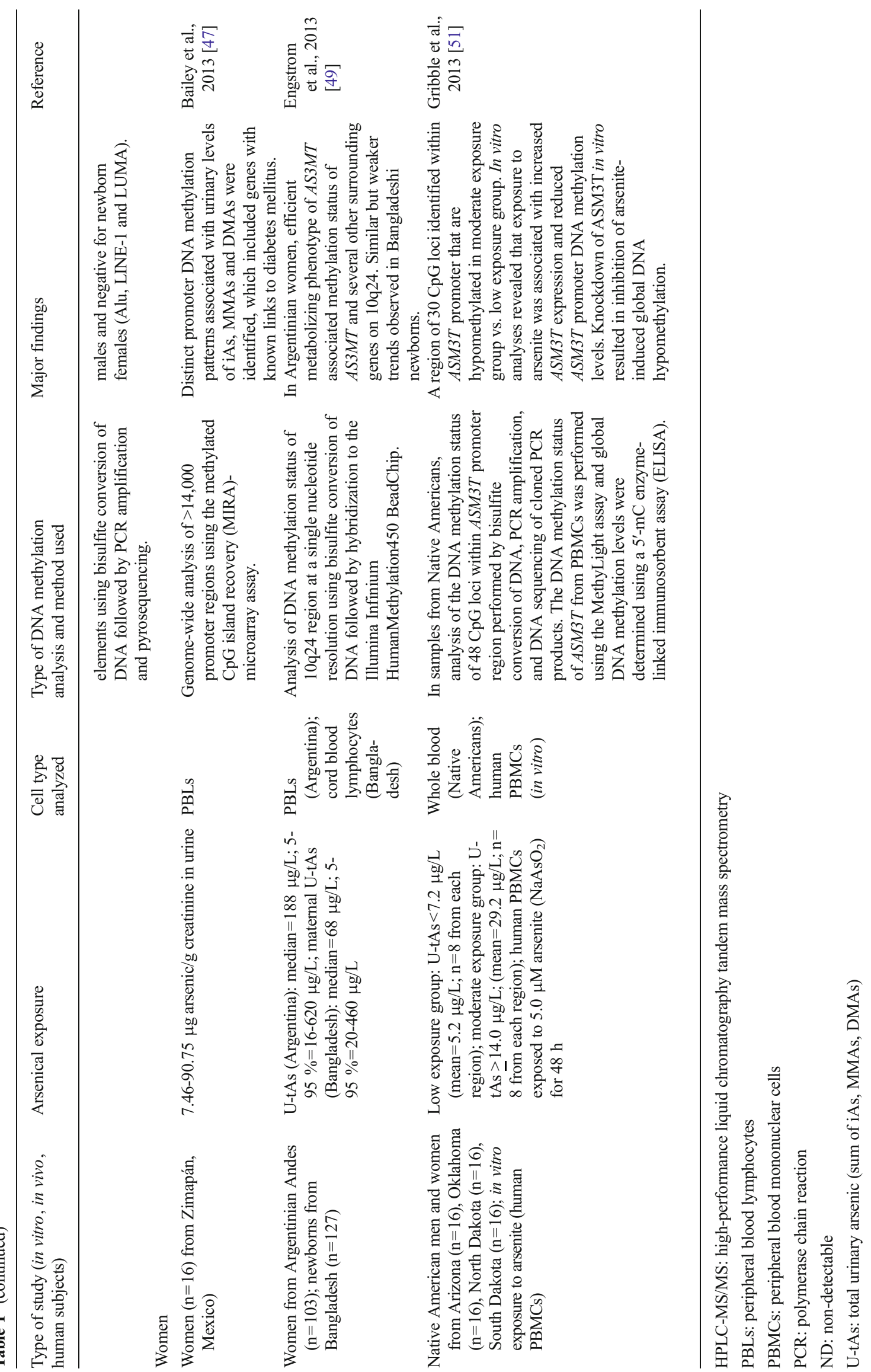


effects on DNA methylation were observed in relationship to maternal U-tAs levels, most of the U-tAs-associated effects were observed in $\mathrm{CpG}$ islands [54•]. Of the $\mathrm{CpG}$ islands tested, $18 \%$ were differentially methylated among newborns associated with different maternal U-tAs levels, and the DNA methylation status of several of these $\mathrm{CpG}$ islands had linear relationships with maternal U-tAs [54•]. Interestingly, a potential sex-dependent relationship was observed between maternal U-tAs levels and global cord blood DNA methylation in Bangladeshi newborns [55•]. While there was a positive relationship between maternal U-tAs levels and global cord blood DNA methylation in all newborns in the study, when the newborns were divided by sex, this relationship was positive for males and negative for females. If the identified changes in DNA methylation levels are, indeed, functional and impact gene/protein expression, these alterations may influence differential susceptibility to iAs-induced disease in males and females, a feature observed in various cohorts exposed to iAs. Taken together, these studies are informative, as they reveal that even low to moderate levels of iAs exposure can impact the DNA methylation landscape. They also highlight the intriguing finding that some of these effects may be sexdependent and suggest an association between iAs metabolism and DNA methylation patterns.

There are a few caveats to consider when interpreting the existing body of literature related to arsenical-associated changes in DNA methylation patterning. For example, although PBL DNA methylation profiles may serve as useful biomarkers for iAs exposure, disease state, and/or disease susceptibility, it is currently unknown whether PBL DNA methylation profiles reflect those that would be present in target cells of iAs toxicity such as liver, lung, and bladder. In addition, it is important to note that multiple white blood cell types are present in PBL preparations, and these cell populations may shift upon exposure to iAs or due to other environmental factors. It is also unknown if the gene-specific DNA methylation changes correspond to changes in mRNA or protein levels of their genes. Additionally, the relationship between DNA methylation and gene expression is more complex than often considered, where increased DNA methylation may be associated with increased gene expression. There is currently limited information on the stability of the DNA methylation changes over time. Also, in the case of prenatal iAs exposure, the studies presented thus far do not investigate true transgenerational effects, as iAs can readily pass through the placenta [56, 57], and thus warrant an assessment of transgenerational effects of various iAs-associated phenotypes that persist to the F3 generation and beyond. It is important to note, therefore, that while the available studies serve as a critical foundation for understanding the association between iAs exposure and the epigenome, future studies will be necessary to examine the relationship between these alterations and the development of iAs-associated disease.

\section{Arsenic and histone post-translational modifications (PTMs)}

Histones play an essential role in the compaction and organization of the genome and in the regulation of gene activity. Histones comprise the protein component of the nucleosome, which is the fundamental repeating unit of chromatin. The nucleosome contains $\sim 146$ bp of DNA wrapped around a histone octamer comprising two copies each of the four core histones H2A, H2B, H3, and H4 [58]. Each of the four core histones contains unstructured amino-terminal (N-terminal) and carboxy-terminal (C-terminal) tails, and specific amino acids within those tails are targets of post-translational modifications (PTMs) [59]. Each histone may have multiple PTMs, which collectively comprise the "histone code" of a region and therefore influence its transcriptional competency via effects on nucleosome structure/positioning and by acting as protein-binding targets [60]. While multiple PTMs have been identified, those that are the most extensively studied and best understood in the context of transcriptional competency are histone phosphorylation, methylation, acetylation, and ubiquitination [61].

Most of the investigations into the effects of arsenicals on histone PTMs have been performed in vitro, in which changes in both global and gene-specific histone PTM patterns have been observed (as reviewed in [22]). Of note, several of these gene-specific histone PTM alterations have identified a relationship between arsenic exposure, the acquisition of targeted promoter histone PTM marks and DNA methylation patterns, and transcriptional competency. In at least one example, altered histone PTMs and the altered expression of a particular gene were associated with the toxic effects of arsenic. For instance, the arsenical-mediated malignant transformation of a human urothelial cell line was associated with aberrant promoter histone PTMs and overexpression of a key gene, namely wingless-type MMTV integration site family, member $5 \mathrm{~A}$ (WNT5A), which is essential for the maintenance of the malignant phenotype [62].

As observed in the case of DNA methylation, recent years have witnessed an increase in the number of studies that have examined histone PTMs in iAs-exposed populations (Table 2). For instance, a positive association was observed among steel workers exposed to inhalable metal-rich particles between arsenic and nickel exposure and global PBL histone H3 lysine 4 dimethylation (H3K4me2) and histone 3 lysine 9 acetylation (H3K9ac) in PBLs [63]. A study of iAs-exposed individuals in Bangladesh revealed that some arsenic-associated changes in histone PTMs were common across all individuals, whereas others were sex-specific [64•]. For instance, U-tAs levels were positively associated with the transcription-restrictive PTM histone 3 lysine 9 dimethylation (H3K9me2) and negatively associated with the transcription-permissive histone PTM histone 3 lysine 9 acetylation (H3K9ac) in the PBLs of both men 


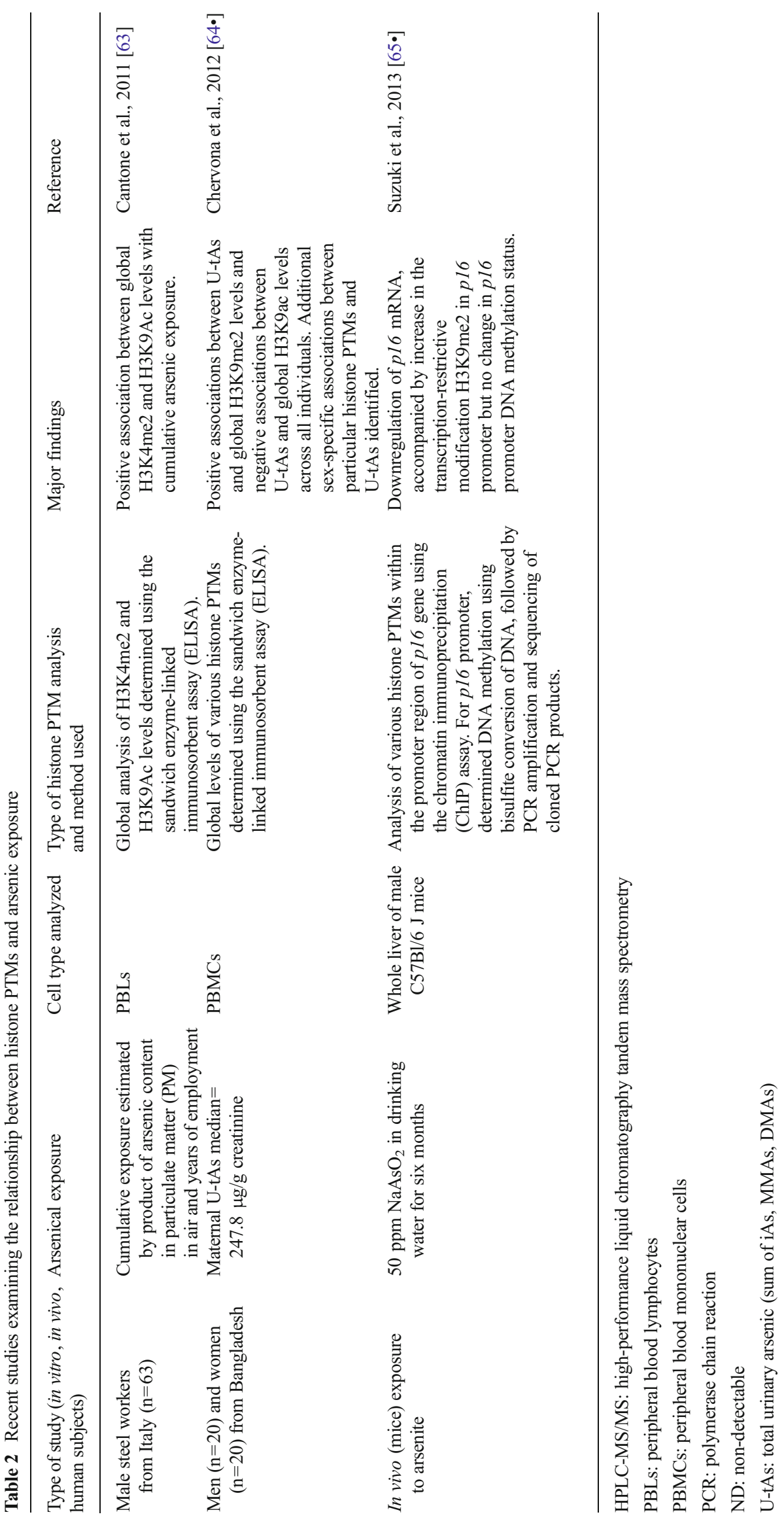


and women. The authors point out that the positive association of $\mathrm{H} 3 \mathrm{~K} 9 \mathrm{me} 2$ with U-tAs is particularly interesting in light of the DNA hypermethylation of LINE-1 elements in iAsexposed mother-child pairs in Bangladesh [53], as $\mathrm{H} 3 \mathrm{~K} 9 \mathrm{me} 2$ is a marker for DNA methylation. Therefore, data from these two studies are consistent with arsenic-associated epigenetic effects that may favor overall transcriptional repression [64•]. However, in addition to trends observed for specific histone PTMs in both men and women, four other histone PTMs were identified that exhibited sex-dependent associations with levels of iAs in drinking water [64•].

One study in mice indicated that changes in promoter PTMs are not always associated with changes in DNA methylation patterns. Long-term (6 months) exposure to iAs resulted in the downregulation of $p 16$ mRNA in the livers of C57BL/6 mice, which was accompanied by an increase of the transcription-restrictive PTM H3K9me2 in the p16 promoter region but no change in promoter DNA methylation status [65•].

Together, these recent studies demonstrated that exposure to iAs is associated with genome-wide changes in histone PTMs in animals and humans and that some of these effects in humans may be sex-specific. Importantly, mechanistic in vitro and in vivo studies have provided important groundwork for studying the relationship between iAs exposure, changes in histone PTMs, and potentially toxic effects, such as the transcriptional modulation of genes implicated in disease development (e.g., tumor suppressor $p 16$ ). The relationship between these events and iAs-associated disease development must be addressed in future work. In addition, the studies highlighted here underscore the need to examine the interplay between multiple epigenetic components that likely work in unison to mediate the toxic effects of arsenicals.

\section{Arsenic and microRNAs (miRNAs)}

miRNAs are non-coding RNAs 21-24 nucleotides in length that are best characterized as regulators of gene activity at the post-transcriptional level, although they have been shown to regulate gene expression at the transcriptional level as well $[66,67]$. At the post-transcriptional level, miRNAs bind to the 3' untranslated region (3' UTR) of mRNAs, which can alter mRNA function by interfering with mRNA translation into protein or by targeting mRNAs for degradation [68]. The regulation of miRNA expression is complex, as miRNA levels are controlled not only at the transcriptional level but also post-transcriptionally, where they undergo multiple processing steps to yield mature miRNAs [69]. Approximately 2000 mature miRNAs have been identified in humans [70], and by analyzing conserved miRNA binding sites within the 3' UTR, it is believed that $>60 \%$ of protein-encoding human genes are targets of miRNAs [71].
The effects of iAs on miRNA levels have been relatively unexplored in comparison to other epigenetic modifications, particularly as they relate to chronic disease development in humans (as reviewed in [22]). Previous reviews have highlighted that most studies of the effects of arsenical exposure on miRNAs have been performed in vitro, and few have investigated the functional consequences of these perturbations [22, 23]. In recent years, in vitro studies have continued to predominate, but many are mechanistic in nature, in which miRNAs have been implicated in mediating various toxic effects of arsenicals (Table 3 ).

Some of these studies have focused on miRNAs as mediators of the apoptotic effects of arsenic trioxide (ATO) [72, 73], a chemotherapeutic agent used in the treatment of relapsed or refractory acute promyelocytic leukemia [74]. Other studies have indicated miRNAs may play roles in the diverse toxic effects of iAs. For instance, miR-9 and miR-181b were implicated as promoting abnormal angiogenesis in iAsexposed chick embryos [75], and specific miRNAs have been implicated in iAs-mediated carcinogenesis. As an example, the reduction of miR-200b was shown to be essential for the iAs-mediated malignant transformation of TP53-deficient human bronchial epithelial cells [76]. Interestingly, the iAsmediated miR-200b reduction was accompanied by increased methylation of the miR-200 promoter [76], highlighting the potential interplay between multiple forms of epigenetic regulation on gene expression. IAs was also found to induce miR190 in a dose-dependent manner in a human bronchial cell line, in which miR-190 overexpression was shown to enhance malignant characteristics [77]. This study also identified a target of miR-190, namely mRNA of the PH domain leucine-rich repeat protein phosphatase (PHLPP) gene, which has known tumor suppressor functions. Another study identified the upregulation of miR-21 as an important event in the iAs-mediated transformation of human embryo lung fibroblast cells, which was dependent on the activation of ROSsensitive pathways involving extracellular signal-regulated kinase mitogen-activated protein kinase (ERK/MAPK) and transcription factor nuclear factor kappa-light-chain-enhancer of activated B cells (NF-kB) [78•].

Together, these studies indicate that miRNAs may be involved in mediating multiple toxic effects of arsenic species, including the modulation of pathways that control cell growth, differentiation, and apoptosis. The studies also provide insight regarding the involvement of epigenetic modifications and cellular signaling pathways in the modulation of miRNA levels in response to iAs exposure. Future research could elucidate the mechanisms by which iAs can perturb miRNA expression levels and assess whether such expression is altered in biological samples from humans. As with the other forms of epigenetic modifications, it will be critical to confirm the functional consequences of changes in the expression of miRNAs on the expression of their transcriptional targets and 


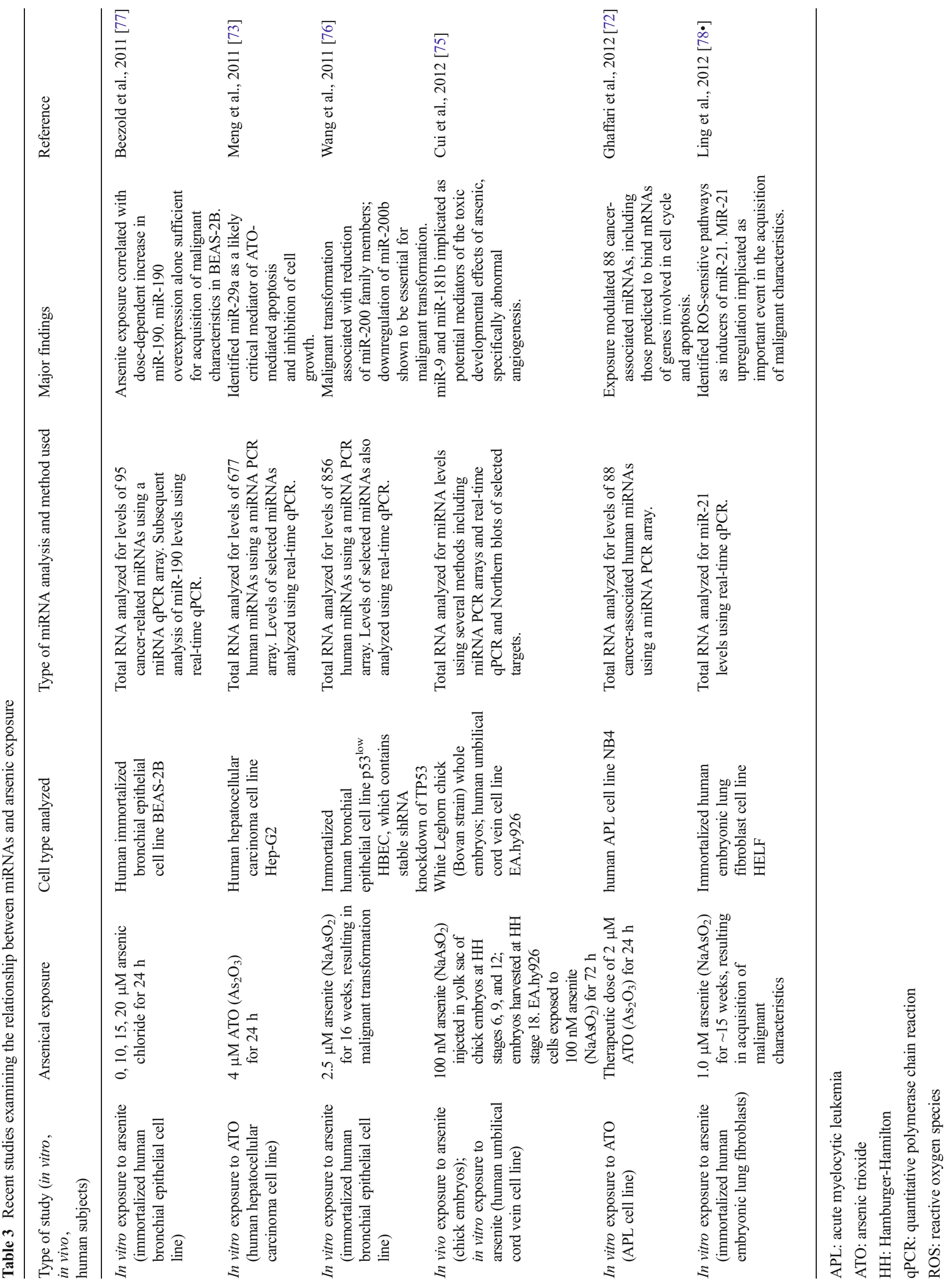


to determine whether these changes ultimately influence protein abundance and function.

\section{Conclusions and future directions}

In summary, current literature clearly indicates that arsenic exposure is associated with changes that include altered patterns of DNA methylation (both global and gene-specific), modifications of histone PTMs, and altered expression levels of miRNAs. Importantly, answers to some key questions remain unknown: (1) What is the relationship of the epigenetic changes to disease?-The significance of these arsenicassociated epigenetic effects on human disease development is likely dependent on a number of factors, including genotype, combined exposures to other contaminants, and other environmental influences such as nutrition; (2) How do the epigenetic changes influence disease directly?-While many associations have been established, it is largely unknown if there is a causal relationship between the epigenetic changes and arsenic-associated disease development, as well as the mechanisms by which these changes cause disease; (3) How stable are the epigenetic changes and are they functional?-It is important to consider that some epigenetic changes may be adaptive or stochastic responses that do not promote disease development. Likewise, global and/or gene-specific changes in DNA methylation patterning and histone PTMs may not be reflective of functional changes that are manifested at the protein level. Of note, it is important to consider that several major research trends have emerged in recent years. For instance, the DNA methylation studies were almost exclusively conducted on samples from human populations, whereas the miRNA studies were dominated by in vitro studies. These trends reveal gaps in research that must be closed in order to fully understand the molecular mechanisms that drive arsenical-associated epigenetic alterations, the consistency of these alterations across experimental platforms and study type (e.g., in vitro studies, in vivo studies, human studies), and ultimately, the relevance of these alterations to altered disease risk in exposed populations. Lastly, it will be increasingly important to examine the effects of arsenicals at a systemswide level, where the impacts of both epigenetic and nonepigenetic alterations are examined. It is likely that the true biological picture of the epigenetic contributions to the mechanism of iAs-induced disease represents the complex interplay of a multitude of signaling events within the cell.

Acknowledgments This work was supported in part by the NIEHS (T32 ES007018, ES019315, ES005948, and ES010126).

\section{Compliance with Ethics Guidelines}

Conflict of Interest Kathryn A. Bailey and Rebecca C. Fry declare that they have no conflicts of interest.
Human and Animal Rights and Informed Consent This article does not contain any studies with human or animal subjects performed by any of the authors.

Open Access This article is distributed under the terms of the Creative Commons Attribution License which permits any use, distribution, and reproduction in any medium, provided the original author(s) and the source are credited.

\section{References}

Papers of particular interest, published recently, have been highlighted as:

- Of importance

1. Nordstrom DK. Public health Worldwide occurrences of arsenic in ground water. Science. 2002;296(5576):2143-5.

2. WHO, WHO, World Health Organization. Guidelines for drinking water quality. Geneva: WHO Press; 2006.

3. Uddin R, Huda NH. Arsenic poisoning in bangladesh. Oman Med J. 2011;26(3):207.

4. Sengupta SR, Das NK, Datta PK. Pathogenesis, clinical features and pathology of chronic arsenicosis. Indian J Dermatol Venereol Leprol. 2008;74(6):559-70.

5. Rahman MM, Ng JC, Naidu R. Chronic exposure of arsenic via drinking water and its adverse health impacts on humans. Environ Geochem Health. 2009;31 Suppl 1:189-200.

6. Tseng $\mathrm{CH}$. Arsenic methylation, urinary arsenic metabolites and human diseases: current perspective. J Environ Sci Health $\mathrm{C}$ Environ Carcinog Ecotoxicol Rev. 2007;25(1):1-22.

7. Jomova $\mathrm{K}$ et al. Arsenic: toxicity, oxidative stress and human disease. J Appl Toxicol. 2011;31(2):95-107.

8. Inbar-Feigenberg $\mathrm{M}$ et al. Basic concepts of epigenetics. Fertil Steril. 2013;99(3):607-15.

9. Barros SP, Offenbacher S. Epigenetics: connecting environment and genotype to phenotype and disease. J Dent Res. 2009;88(5): 400-8.

10. Dolinoy DC, Jirtle RL. Environmental epigenomics in human health and disease. Environ Mol Mutagen. 2008;49(1):4-8.

11. Baccarelli A, Bollati V. Epigenetics and environmental chemicals. Curr Opin Pediatr. 2009;21(2):243-51.

12. Portela A, Esteller M. Epigenetic modifications and human disease. Nat Biotechnol. 2010;28(10):1057-68.

13. Bollati V, Baccarelli A. Environmental epigenetics. Heredity (Edinb). 2010;105(1):105-12.

14. Farzan SF, Karagas MR, and Chen Y. In utero and early life arsenic exposure in relation to long-term health and disease. Toxicol Appl Pharmacol. 2013;272(2):384-390.

15. Waalkes MP et al. Transplacental carcinogenicity of inorganic arsenic in the drinking water: induction of hepatic, ovarian, pulmonary, and adrenal tumors in mice. Toxicol Appl Pharmacol. 2003;186(1):7-17.

16. Smith AH et al. Mortality in young adults following in utero and childhood exposure to arsenic in drinking water. Environ Health Perspect. 2012;120(11):1527-31.

17. Waalkes MP et al. Estrogen signaling in livers of male mice with hepatocellular carcinoma induced by exposure to arsenic in utero. $\mathrm{J}$ Natl Cancer Inst. 2004;96(6):466-74.

18. Xie $\mathrm{Y}$ et al. Aberrant DNA methylation and gene expression in livers of newborn mice transplacentally exposed to a hepatocarcinogenic dose of inorganic arsenic. Toxicology. 2007;236(1-2):7-15. 
19. Jirtle RL, Skinner MK. Environmental epigenomics and disease susceptibility. Nat Rev Genet. 2007;8(4):253-62.

20. Dolinoy DC, Weidman JR, Jirtle RL. Epigenetic gene regulation: linking early developmental environment to adult disease. Reprod Toxicol. 2007;23(3):297-307.

21. Cropley JE et al. Germ-line epigenetic modification of the murine A vy allele by nutritional supplementation. Proc Natl Acad Sci U S A. 2006;103(46):17308-12.

22. Bailey KA, Fry RC. In: Sahu SC, editor. Arsenic-induced Changes to the Epigenome, in Toxicology and Epigenetics. Chichester: John Wiley \& Sons, Ltd; 2012.

23. Ren $\mathrm{X}$ et al. An emerging role for epigenetic dysregulation in arsenic toxicity and carcinogenesis. Environ Health Perspect. 2011;119(1):11-9.

24. Reichard JF, Puga A. Effects of arsenic exposure on DNA methylation and epigenetic gene regulation. Epigenomics. 2010;2(1):87104.

25. Arrigo AP. Acetylation and methylation patterns of core histones are modified after heat or arsenite treatment of Drosophila tissue culture cells. Nucleic Acids Res. 1983;11(5):1389-404.

26. Camato R, Tanguay RM. Changes in the methylation pattern of core histones during heat-shock in Drosophila cells. EMBO J. 1982;1(12):1529-32.

27. Zhao CQ et al. Association of arsenic-induced malignant transformation with DNA hypomethylation and aberrant gene expression. Proc Natl Acad Sci U S A. 1997;94(20):10907-12.

28. Mass MJ, Wang L. Arsenic alters cytosine methylation patterns of the promoter of the tumor suppressor gene p53 in human lung cells: a model for a mechanism of carcinogenesis. Mutat Res. 1997;386(3):263-77.

29. Bird A. The essentials of DNA methylation. Cell. 1992;70(1):5-8.

30. Irizarry RA et al. The human colon cancer methylome shows similar hypo- and hypermethylation at conserved tissue-specific CpG island shores. Nat Genet. 2009;41(2):178-86.

31. Gardiner-Garden M, Frommer M. CpG islands in vertebrate genomes. J Mol Biol. 1987;196(2):261-82.

32. Haluskova J. Epigenetic studies in human diseases. Folia biologica. 2010;56(3):83-96.

33. van Eijk KR et al. Genetic analysis of DNA methylation and gene expression levels in whole blood of healthy human subjects. BMC Genomics. 2012;13:636.

34. Boellmann F et al. Genome-wide analysis of DNA methylation and gene expression changes in the mouse lung following subchronic arsenate exposure. Toxicol Sci. 2010;117(2):404-17.

35. Wilson AS, Power BE, Molloy PL. DNA hypomethylation and human diseases. Biochim Biophys Acta. 2007;1775(1):138-62.

36. Benbrahim-Tallaa L et al. Molecular events associated with arsenicinduced malignant transformation of human prostatic epithelial cells: aberrant genomic DNA methylation and K-ras oncogene activation. Toxicol Appl Pharmacol. 2005;206(3):288-98.

37. Coppin JF, Qu W, Waalkes MP. Interplay between cellular methyl metabolism and adaptive efflux during oncogenic transformation from chronic arsenic exposure in human cells. J Biol Chem. 2008;283(28):19342-50.

38. Dolinoy DC et al. Maternal genistein alters coat color and protects Avy mouse offspring from obesity by modifying the fetal epigenome. Environ Health Perspect. 2006;114(4):567-72.

39. Waterland RA, Jirtle RL. Early nutrition, epigenetic changes at transposons and imprinted genes, and enhanced susceptibility to adult chronic diseases. Nutrition. 2004;20(1):63-8.

40. Perera F et al. Relation of DNA methylation of 5'-CpG island of ACSL3 to transplacental exposure to airborne polycyclic aromatic hydrocarbons and childhood asthma. PLoS One. 2009;4(2):e4488.

41. Prins GS et al. Developmental exposure to bisphenol A increases prostate cancer susceptibility in adult rats: epigenetic mode of action is implicated. Fertil Steril. 2008;89(2 Suppl):e41.
42. Skinner MK, Manikkam M, Guerrero-Bosagna C. Epigenetic transgenerational actions of endocrine disruptors. Reprod Toxicol. 2011;31(3):337-43.

43. Thomas DJ, Styblo M, Lin S. The cellular metabolism and systemic toxicity of arsenic. Toxicol Appl Pharmacol. 2001;176(2):127-44.

44. Vahter M. Mechanisms of arsenic biotransformation. Toxicology. 2002;181-182:211-7.

45. Drobna Z, Styblo M, and Thomas DJ, An Overview of Arsenic Metabolism and Toxicity, in Current Protocols in Toxicology, Supplement 42: Techniques for Analysis of Chemical Biotransformation. 2009, John Wiley and Sons.

46. Smeester L et al. Epigenetic changes in individuals with arsenicosis. Chem Res Toxicol. 2011;24(2):165-7. Using a genome-wide, gene-specific approach, this study identified multiple genes with differentially methylated promoters in individuals with arsenicassociated skin lesions.

47. Bailey KA et al. Arsenic and the epigenome: interindividual differences in arsenic metabolism related to distinct patterns of DNA methylation. J Biochem Mol Toxicol. 2013;27(2):106-15.

48. Sanders AP, et al. Cadmium exposure and the epigenome: Exposure-associated patterns of DNA methylation in leukocytes from mother-baby pairs. Epigenetics. 2013; 9(2).

49. Engstrom KS et al. Efficient arsenic metabolism-the AS3MT haplotype is associated with DNA methylation and expression of multiple genes around AS3MT. PLoS One. 2013;8(1):e53732.

50. Hossain MB et al. Environmental arsenic exposure and DNA methylation of the tumor suppressor gene p16 and the DNA repair gene MLH1: effect of arsenic metabolism and genotype. Metallomics. 2012;4(11):1167-75. This study identified distinct PBL DNA methylation patterns associated with arsenic (+3 oxidation state) methyltransferase AS3MT haplotype and levels of urinary arsenical metabolites in an exposed population.

51. Gribble MO, et al. Differential methylation of the arsenic (III) methyltransferase promoter according to arsenic exposure. Arch Toxicol. 2013

52. Intarasunanont $\mathrm{P}$ et al. Effects of arsenic exposure on DNA methylation in cord blood samples from newborn babies and in a human lymphoblast cell line. Environ Health. 2012;11:31.

53. Kile ML et al. Prenatal arsenic exposure and DNA methylation in maternal and umbilical cord blood leukocytes. Environ Health Perspect. 2012;120(7):1061-6.

54. Koestler DC et al. Differential DNA methylation in umbilical cord blood of infants exposed to low levels of arsenic in utero. Environ Health Perspect. 2013;121(8):971-7. Using a genome-wide approach, this study revealed that relatively low levels of prenatal arsenic exposure are associated with changes in the DNA methylome in newborn cord blood.

55. Pilsner JR et al. Influence of prenatal arsenic exposure and newborn sex on global methylation of cord blood DNA. PLoS One. 2012;7(5):e37147. This study revealed that prenatal arsenic exposure is associated with global changes in DNA methylation status in newborn cord blood, possibly in a sex-dependent manner.

56. Vahter M. Health effects of early life exposure to arsenic. Basic Clin Pharmacol Toxicol. 2008;102(2):204-11.

57. Concha $\mathrm{G}$ et al. Exposure to inorganic arsenic metabolites during early human development. Toxicol Sci. 1998;44(2):185-90.

58. Luger $\mathrm{K}$ et al. Crystal structure of the nucleosome core particle at 2.8 A resolution. Nature. 1997;389(6648):251-60.

59. Luger K, Richmond TJ. The histone tails of the nucleosome. Curr Opin Gen Dev. 1998;8(2):140-6.

60. Jenuwein T, Allis CD. Translating the histone code. Science. 2001;293(5532):1074-80.

61. Peterson CL, Laniel MA. Histones and histone modifications. Curr Biol: CB. 2004;14(14):R546-51. 
62. Jensen TJ et al. Epigenetic mediated transcriptional activation of WNT5A participates in arsenical-associated malignant transformation. Toxicol Appl Pharmacol. 2009;235(1):39-46.

63. Cantone L, et al. Inhalable Metal-rich Air Particles and Histone H3K4 Dimethylation and H3K9 Acetylation in a Cross-sectional Study of Steel Workers. Environmental health perspectives. 2011.

64. Chervona Y et al. Associations between arsenic exposure and global posttranslational histone modifications among adults in Bangladesh. Cancer Epidemiol Biomarkers Prev. 2012;21(12): 2252-60. This study identified global histone PTMs in the PBLs of arsenic-exposed humans, some of which were sex-specific.

65. Suzuki T, Nohara K. Long-term arsenic exposure induces histone H3 Lys9 dimethylation without altering DNA methylation in the promoter region of $\mathrm{p} 16(\mathrm{INK} 4 \mathrm{a})$ and down-regulates its expression in the liver of mice. J Appl Toxicol. 2013;33(9):951-8. This study of long-term arsenic exposure in mice indicated that changes in expression of genes may be associated with alterations in promoter histone PTMs but no change in DNA methylation status.

66. Tan Y et al. Transcriptional inhibiton of Hoxd4 expression by miRNA-10a in human breast cancer cells. BMC Mol Biol. 2009;10:12.

67. He L, Hannon GJ. MicroRNAs: small RNAs with a big role in gene regulation Nature reviews. Genetics. 2004;5(7):522-31.

68. Hudder A, Novak RF. miRNAs: effectors of environmental influences on gene expression and disease. Toxicol Sci. 2008;103(2): $228-40$.

69. Shukla GC, Singh J, Barik S. MicroRNAs: Processing, Maturation, Target Recognition and Regulatory Functions. Mol Cell Pharmacol. 2011;3(3):83-92.
70. Kozomara A and Griffiths-Jones S. miRBase: integrating microRNA annotation and deep-sequencing data. Nucleic Acids Res. 2011; 39(Database issue): D152-7.

71. Friedman RC et al. Most mammalian mRNAs are conserved targets of microRNAs. Genome Res. 2009;19(1):92-105.

72. Ghaffari SH et al. Alteration in miRNA gene expression pattern in acute promyelocytic leukemia cell induced by arsenic trioxide: a possible mechanism to explain arsenic multi-target action. Tumour Biol. 2012;33(1):157-72.

73. Meng $\mathrm{XZ}$ et al. microRNA expression alteration after arsenic trioxide treatment in HepG-2 cells. J Gastroenterol Hepatol. 2011;26(1):186-93.

74. Soignet SL et al. United States multicenter study of arsenic trioxide in relapsed acute promyelocytic leukemia. J Clin Oncol. 2001;19(18):3852-60.

75. Cui $\mathrm{Y}$ et al. MicroRNA-181b and microRNA-9 mediate arsenicinduced angiogenesis via NRP1. J Cell Physiol. 2012;227(2):772-83.

76. Wang $Z$ et al. Reversal and prevention of arsenic-induced human bronchial epithelial cell malignant transformation by microRNA200b. Toxicol Sci. 2011;121(1):110-22.

77. Beezhold K et al. miR-190-mediated downregulation of PHLPP contributes to arsenic-induced Akt activation and carcinogenesis. Toxicol Sci. 2011;123(2):411-20.

78. Ling $\mathrm{M}$ et al. Regulation of miRNA-21 by reactive oxygen speciesactivated ERK/NF-kappaB in arsenite-induced cell transformation. Free Radic Biol Med. 2012;52(9):1508-18. This study identified relationships between activated cellular signaling pathways, aberrant miRNA expression and acquisition of malignant characteristics during arsenic-mediated transformation of human embryonic lung fibroblasts. 